\title{
Gratitude Texting Touches the Heart: Challenge/Threat Cardiovascular Responses to Gratitude Expression Predict Self-initiation of Gratitude Interventions in Daily Life
}

\author{
Jolanta Enko ${ }^{1}$ D $\cdot$ Maciej Behnke ${ }^{1} \cdot$ Martyna Dziekan $^{1} \cdot$ Michał Kosakowski $^{1}$. \\ Lukasz D. Kaczmarek ${ }^{1}$
}

Published online: 20 January 2020

(C) The Author(s) 2020

\begin{abstract}
Gratitude-based interventions are effective in facilitating positive relationships and increasing life satisfaction. However, for some individuals (e.g., with high levels of depression and low trait-gratitude) gratitude expression is threatening and rarely undertaken spontaneously. In this study, we expected to replicate this gratitude expression threat effect. Moreover, we aimed to understand psychophysiological mechanisms of this effect by accounting for cognitive, motivational, and physiological responses to gratitude expression in line with the biopsychosocial model of challenge and threat. One hundred ninety-six students (51\% women) between the ages of 18 and 31 years old $(M=21.20, S D=2.08)$ reported personality measures and completed a laboratory session where they expressed gratitude via text messages after reporting evaluation and motivation towards the task. Their cardiovascular reactivity was monitored continuously. After the session, participants were invited to continue a gratitude intervention for the next three weeks. We found that individuals with higher depression and lower trait-gratitude were less likely to initiate gratitude intervention. This effect was mediated by a cardiovascular marker of threat (total peripheral resistance) that inhibited motivation and behavior. In summary, we replicated and provided further evidence for the role of personality traits in predicting aversive responses to gratitude expression via interventions. These findings contribute to the person-activity fit recommendations.
\end{abstract}

Keywords Gratitude · Cardiovascular activity · Positive psychological interventions · Biopsychosocial model of challenge and threat $\cdot$ Challenge $\cdot$ Threat

Jolanta Enko

jenko@amu.edu.pl

1 Faculty of Psychology and Cognitive Science, Adam Mickiewicz University, 89 Szamarzewskiego Street, 60-568 Poznań, Poland 


\section{Introduction}

Gratitude assists individuals in the maintenance of supportive social bonds and greater individual quality of life (Emmons and McCullough 2003; Seligman et al. 2005; Froh et al. 2009). Grateful experience and grateful interpersonal behaviors can be exercised and enhanced via interventions. Several brief gratitude-based cognitive exercises have been proposed that are effective in increasing individual well-being and promoting healthier social relationships (Sin and Lyubomirsky 2009; Bolier et al. 2013; Chakhssi et al. 2018; Hendriks et al. 2018a, b). Interventions that enhance expression of gratitude towards other individuals (e.g., writing brief thankful e-mails to benefactors), are among the most effective positive psychological interventions relative to other interventions (Seligman et al. 2005) and their effects extend to relational benefits (Lambert et al. 2010).

Despite being among the most effective, interpersonal gratitude interventions are among the most demanding (Huffman et al. 2014; Kaczmarek et al. 2015). Communicating gratitude is likely to induce mixed emotions because grateful individuals implicitly admit that they are dependent on others (Wood et al. 2010). They may also be unsure about the benefactors' response to their gratitude expression. For instance, the Letter of Gratitude intervention-where gratitude is communicated to the benefactor in a written and oral form-is rarely preferred relative to Gratitude Journaling - an intervention based on individual contemplation of grateful feelings (Kaczmarek et al. 2015). This indicates the need to put more emphasis on person-activity fit as a critical aspect of assigning interventions to individuals based on their personality traits (Lyubomirsky and Layous 2013; Senf and Liau 2013).

We offer a novel psychophysiological lab-based approach towards testing the personactivity fit. With the majority of positive intervention studies using self-reports, we aimed to introduce physiological measures into positive intervention literature. The biopsychosocial model of challenge and threat (Blascovich and Mendes 2000) offers an adequate framework to interpret physiological reactivity related to the intervention. Using a multilayer approach, we aimed to test how cognitive (evaluations, motivations, and intentions) and physiological (cardiovascular) responses to lab-based social tasks, predict subsequent daily actions. The positive psychological interventions literature (e.g., Lyubomirsky et al. 2011) needs more comprehensive and integrative models that explain why positive interventions have high efficacy but low effectiveness (Hone et al. 2015), i.e., why individuals have problems with initiation and completion of these interventions despite their documented positive potential. The psychophysiological perspective might be one way to address this problem by reading uncontrolled bodily responses that might influence motivations and actions. Finally, this line of studies is also likely to contribute to a more comprehensive evaluation of the biopsychosocial model of challenge and threat that needs more diverse areas of application in testing its validity in predicting favorable behavioral outcomes (Behnke and Kaczmarek 2018).

\subsection{The Costs and Benefits of Gratitude Expression}

Interpersonal expression of gratitude via structured interventions is of particular interest to the science of well-being, as it offers relational benefits, such as increased communal strength, in addition to personal benefits (Lambert et al. 2010). Nurturing social relationships is the most meaningful happiness-increasing activity in everyday life (Parks et al. 2012). Sharing grateful feelings might be one way to enhance social bonds. In 
previous studies, self-reported effort used to perform a positive intervention predicted greater gains from the interventions (Lyubomirsky et al. 2011). This points to a problem that these potentially beneficial interventions are difficult to initiate and maintain (Kaczmarek et al. 2015). Examination of physiological indexes of intervention-related motivation and effort can help explain conditions for interventions' efficacy.

Individuals who are capable of mobilizing sufficient levels of effort to engage in a positive intervention report greater gains in well-being than those who perform interventions superficially (Sin and Lyubomirsky 2009; Lyubomirsky et al. 2011). Research has begun to identify personality characteristics (Kaczmarek et al. 2013), and how they interact with motivational belief systems about the intervention (Kaczmarek et al. 2014a), to predict self-initiation of a gratitude intervention.

Interpersonal gratitude interventions, such as communicating gratitude via text messages, e-mails, or letters (Lambert et al. 2010; Kaczmarek et al. 2015) are among the most effective in increasing well-being (Seligman et al. 2005; Huffman et al. 2014). At the same time, they are the least preferred interventions, presumably because they are demanding (Parks et al. 2012). Three motivational components derived from the theory of planned behavior (Ajzen 1991, 2011) have been shown to inform the decision to self-initiate a gratitude intervention (Kaczmarek et al. 2014a, b, 2015). These are the attitude towards the behavior (utility beliefs), beliefs about the approval of the action by significant others (social norm beliefs), and beliefs about the personal capacity to handle demands of the intervention enactment (self-control beliefs) (Ajzen 1991, 2011). All three components predict intention to perform the gratitude intervention and intention predicts actual intervention self-initiation. Compared to less motivated peers, individuals with strong intentions to change are twice as likely to perform an intervention (Kaczmarek et al. 2013, 2014a).

Forced gratitude expression can be excessively demanding for some individuals, causing low effectiveness of interventions. For example, while meta-analysis by Sin and Lyubomirsky (2009) showed that clinical groups (e.g., individuals with depression) benefit from positive interventions, individuals from such groups might lack the motivational resources necessary to initiate and continue an intervention on their own (Kaczmarek et al. 2013). This might explain why interventions are more effective when applied in psychotherapy settings that are likely to provide sufficient motivational assistance compared to self-help interventions. Moreover, individuals who lack motivation to express gratitude and feel threatened in a situation when gratitude expression is expected are likely to be at the psychological and physiological risk of negative side effects. This is because the physiological pattern of threat (e.g., increased total peripheral resistance) imposes a health risk (Lovallo and Gerin 2003).

Physiological responses to gratitude were researched in previous studies. For instance, a recent study indicated that a gratitude intervention produced more trustful behavior at the expense of stronger physiological reactivity reflected in increased systolic and diastolic blood pressure and respiratory rate (Drążkowski et al. 2017). In another study, a gratitude diary intervention performed regularly for two weeks resulted in decreased diastolic blood pressure that was correlated with changes in well-being (Jackowska et al. 2016). Another study showed a beneficial influence of gratitude on heart rate variability. There were increased parasympathetic heart rate variability responses during gratitude journaling in patients with heart failure (Redwine et al. 2016) and increased buffering against negative effects of rumination on heart rate variability while focusing on the benefits of facing an interpersonal offense (vanOyen Witvliet et al. 2010). 
These psychophysiological studies have established a link between practicing gratitude and health outcomes that have, however, several limitations. First, these findings indicate that despite a general soothing effect of gratitude on physiological processes (Jackowska et al. 2016; Redwine et al. 2016; vanOyen Witvliet et al. 2010), gratitude produces unfavorable effects (e.g., increased arousal) in more specific social situations (Drążkowski et al. 2017). Second, none of these studies accounted for a person-activity fit that might provide further insight into whether there are groups that respond with health-protective and groups that respond with risk-related cardiovascular patterns to gratitude expression. Third, although previous psychophysiological studies have focused on the effects and correlates of gratitude, little is known about how physiological responses to gratitude predict further gratitude-related activity preference and long-term involvement. This is important because individuals benefit more when they self-select (Sin and Lyubomirsky 2009) and actively participate in interventions (Lyubomirsky et al. 2011). Finally, studying the link between gratitude expression and physiological processes might benefit from using an established social psychophysiological framework that informs how specific physiological processes correspond with action-oriented situational evaluations. The biopsychosocial model of challenge and threat offers an adequate framework that integrates cognitive and physiological responses to situational demands and opportunities (Blascovich 2008).

\subsection{Physiological Responses to Challenge and Threat}

Individuals experience challenge and threat states when they are motivated to take goaldirected actions, e.g., learning new skills (Moore et al. 2014). Motivated individuals mobilize the physiological resources that are required for task performance (Mendes and Park 2014). Increased action-oriented motivation leads to activation of sympathetic branch of autonomic nervous system indexed by increased heart rate (HR) and shortened pre-ejection period (PEP, i.e., time in the cardiac cycle from initiation of ventricular depolarization to ejection of blood into the vasculature) (Seery 2011). This fundamental motivational activation is further regulated by evaluations of self and the environment (Blascovich 2008). Individuals evaluate their resources (e.g., skills, knowledge, and abilities) and compare them to task-related demands (e.g., danger, uncertainty, and effort) (Lazarus and Folkman 1984). Individuals that evaluate their resources as meeting or exceeding situational demands face an upcoming situation in the challenge state (Blascovich 2008). In contrast, individuals that evaluate their resources as insufficient to task completion respond to the situation with the threat state (Blascovich 2008).

Evaluating the situation as a challenge or threat produces specific physiological responses indexed by two cardiovascular parameters: cardiac output $(\mathrm{CO}$; the amount of blood ejected from the heart during a minute interval) and total peripheral resistance (TPR; a measure of the total vascular resistance). Evaluating the situation as a challenge leads to the release of adrenaline into the bloodstream, which results in the widening of blood vessels (vasodilatation) and producing lower TPR (Brownley et al. 2000). Decreased TPR protects the vascular system and provides the buffer for increased cardiac action. Challenge cardiovascular response also enhances blood flow (increased $\mathrm{CO}$ ) to the muscles and provides metabolic substrates to the cells required for the upcoming task. Consequently, challenge evaluation and its physiological onset facilitates successful action (Behnke and Kaczmarek 2018; Hase et al. 2018). In contrast, perceiving an upcoming situation as a threat leads to decreased $\mathrm{CO}$ and increased TPR. Appraising the situation as a threat inhibits the release of adrenaline and initiates the release of cortisol that results in narrowing the 
arteries. Threat cardiovascular pattern is related to increased risk of cardiovascular disease (Lovallo and Gerin 2003).

We argue that the biopsychosocial model of challenge and threat can be applied to situations in which individuals are encouraged to take the opportunity to engage in a positive intervention. Such situations are important from the applied science perspective because interventions aim to interrupt spontaneous behavioral patterns and seek to introduce a new active element into the individual thought-action repertoire (Michie et al. 2011). This requires a substantial use of individuals resources such as skills, competencies, preferences, or time. Some individuals are likely to embrace this opportunity, for some it may be neutral, and some may feel reluctant to engage in this kind of activity. These types of responding indicate the range of motivation direction and intensity towards the initiation of gratitude interventions (Huffman et al. 2014; Kaczmarek et al. 2015). Individuals forced to perform an intervention regardless of their resources (a person-activity misfit) are likely to experience the threat state. In contrast, individuals that embrace a novel opportunity for gratitude expression are likely to experience the challenge state. Psychophysiological examination of challenge and threat states allows monitoring the situational person-activity fit continuously, without interrupting task performance; an opportunity that is not feasible for self-reports. Observing physiological responses to a gratitude expression opportunity may provide a new perspective on dynamic processes that are likely to occur in gratituderelated social situations and the implementation of positive gratitude-based interventions.

\subsection{Individual Differences and Gratitude Interventions}

Previous studies found individual differences to be a key factor that differentiates individuals' preferences for gratitude expression. Individuals with high trait gratitude levels and less depressive symptoms were more likely to initiate and continue positive interventions based on gratitude (Kaczmarek et al. 2013, 2015). Moreover, depressed individuals experience a conflict between social pressure to be more grateful and their perceived lower self-efficacy in gratitude expression (Kaczmarek et al. 2014a). This suggests that gratitude expression is likely to be threatening to more depressed individuals and that they might experience stronger threat-related physiological arousal during gratitude expression opportunity. In contrast, individuals who are already more grateful are likely to respond with a challenge-related response due to their increased readiness to respond with gratitude across different contexts (Peterson and Seligman 2004).

Moreover, women are more likely than men to experience and express grateful feelings (Becker and Smenner 1986; Beutel and Marini 1995; Bromnick and Swallow 2001; Gordon et al. 2004; Krause 2006; Ventimiglia 1982). Across several studies, women were more likely than men to self-initiate gratitude interventions (Kaczmarek et al. 2013, 2014a, 2015; Misiak et al. 2015). Women perceive gratitude expression as more socially acceptable and expect to derive more benefits from gratitude expression. For men, the expression of gratitude is perceived as more conflicting (Kashdan et al. 2009). Based on this evidence, we expected that women would report a higher level of gratitude than men, which would, in turn, influence intervention self-initiation.

\subsection{The Present Study}

The present study aimed to introduce into the positive psychological interventions literature the biopsychosocial model of challenge and threat (Blascovich and Mendes 2000). 
We aimed to explain the motivations towards gratitude interventions and their effectiveness by accounting for cardiovascular patterns of challenge and threat. Adding psychophysiological measures to the study of positive psychological interventions provides a more nuanced analysis of experiences among interventions' recipients. For instance, physiological measures are less susceptible to distortions due to self-presentation or memory failings, and allow for continuous and more sensitive monitoring of gratitude expression than selfreported measures (Blascovich et al. 2011). Finally, whereas previous studies on positive psychological interventions accounted for personality traits, cognitive, affective and behavioral outcomes, our perspective introduces a novel biological element that might extend the documented pathways between personality traits and positive behavior.

Based on prior research and theory (Kaczmarek et al. 2013, 2015; Mendes et al. 2007, 2008), we predicted that the challenge-type evaluations and physiological response (higher cardiac output and lower total peripheral resistance) to gratitude expression task would predict stronger motivation (and indirectly intention and behavior in line with theory of planned behavior; Ajzen 1991, 2011) to gratitude intervention self-initiation in daily life. In contrast, threat-type evaluations and physiological responses (no increases in $\mathrm{CO}$ and higher TPR) would predict lower motivation. We expected that reactions to gratitude expression opportunity would be determined by individual differences (e.g. challengeresponse, high trait gratitude, and low depression). Finally, corroborating previous findings, we expected that women would react with a challenge-type response to gratitude interventions more often than men.

\section{Method}

\subsection{Participants}

This study involved 196 students (51\% women) between the ages of 18 and 31 years $(M=21.2 ; S D=2.08)$. Participants were asked to refrain from vigorous physical activity, smoking cigarettes, consuming alcohol and caffeine $2 \mathrm{~h}$ before testing. None of the participants had a history of heart disease. Each participant provided written informed consent and received a cinema voucher for participation in the study. The study was approved by the Institutional Ethics Committee.

\subsection{Study Design}

Upon arrival, volunteers signed the consent and reported their age and gender. Participants were tested individually in a cubicle in front of a PC. They were asked to turn on the airplane mode on their mobile phones. Physiological parameters were recorded continuously during the whole experiment (Table 1). After the experimenter left the testing room, participants received instructions and responded via a PC. First, they reported measures of trait gratitude and depressive symptoms. This period lasted several minutes on average and also served as habituation to the laboratory environment (including the beginning of physiology parameters stabilization, although the physiological recording of that phase was not utilized). Next, individuals proceeded to the main task, where they were asked to send two text messages: one gratitude expression message and one neutral message. The order of sending messages was counterbalanced using a randomization algorithm. Sending each SMS was preceded by (1) 3-min baseline when participants were instructed to relax; (2) 


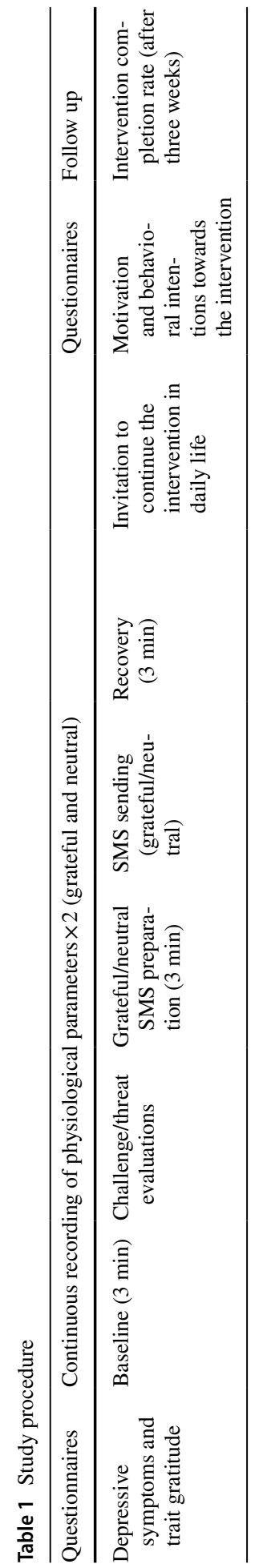


self-reports when participants evaluate upcoming task in terms of challenge and threat; (3) 3-min of text message preparation when participants were asked to think about a person to whom they were grateful for something. After sending the message individuals waited for 3-min as the time needed for physiological recovery. After completing both tasks (sending the grateful and the neutral message), participants were invited to continue the gratitude texting intervention — sending at least three more grateful text messages-for the next three weeks. At the end of the laboratory session, participants reported their motivation (attitudes, social norms, and self-control beliefs) and behavioral intention regarding introduced gratitude texting intervention. Three weeks after the laboratory visit, participants were asked to report the completion of proposed intervention via online form.

\subsection{Measures}

\subsubsection{Challenge/Threat Cardiovascular Markers}

Three cardiovascular indexes were used to measure challenge and threat states: heart rate (HR; in beats per minute); cardiac output (CO; the amount of blood ejected from the heart during a minute interval); total peripheral resistance (TPR; a measure of the total vascular resistance). HR, CO, and TPR were recorded at $1 \mathrm{kHz}$ continuously using Finometer NOVA (Finapres Medical Systems, Netherlands). Finometer uses the volume-clamp method first developed by Penaz (1973) to measure blood pressure. Finger arterial pressure waveforms were recorded with finger cuffs. HR, CO, and TPR data were exported to the Powerlab 16/35 data acquisition system (ADInstruments, New Zealand) and analyzed in LabChart 8.1 (ADInstruments, New Zealand).

\subsubsection{Challenge and Threat Evaluations}

We assessed challenge and threat evaluations with The Cognitive Appraisal Scale (Skinner and Brewer 2002) that consists of two subscales measuring challenge (e.g., "I expect that I will achieve success rather than experience failure") and threat (e.g., "I worry that I will say or do the wrong things"). Participants received explicit instruction to answer the questions regarding sending grateful and neutral text messages. If needed, the sentence structure was modified to pertain to the task of performing gratitude (or neutral) intervention instead of rating overall cognitive style, e.g. "I'm focusing on the positive aspects of this situation", instead of "I tend to focus on the positive aspects of any situation". Participants answered on a 6-point scale ranging from "I strongly disagree" to "I strongly agree". Both subscales had good internal consistency (Cronbach's $\alpha=.83$ for challenge and $\alpha=.92$ for threat).

\subsubsection{Depression Symptoms}

We used the depression scale from the Center for Epidemiological Studies (Radloff 1977) to quantify the frequency of depressive symptoms during the past week (e.g., 'I had crying spells', 'I felt sad'). Participants answered on a 4-point scale ranging from "rarely or none 
of the time (less than 1 day)" to "most or all of the time (5-7 days)". The scale showed a high internal consistency $(\alpha=.90)$.

\subsubsection{Gratitude Trait}

Dispositional gratitude was measured with Gratitude Questionnaire-6 (GQ-6; McCullough et al. 2002), consisting of six items, e.g. "I have so much in my life to be thankful for". Participants answered on a 7-point scale ranging from $1=$ "strongly disagree" to 7 "strongly agree" $(\alpha=.78)$.

\subsubsection{Motivation}

We used four scales to measure motivational components regarding the gratitude expression intervention in daily life: utility beliefs, social norm beliefs, self-control beliefs, and behavioral intention (Francis et al. 2004; Kaczmarek et al. 2015).

\subsubsection{Utility Beliefs}

We assessed participants' attitudes about likely consequences of the proposed tasks, with three 7-point bipolar evaluative adjective scales: "unpleasant-pleasant," "bad-good," and "useless-useful" $(\alpha=.76)$.

\subsubsection{Social Norm Beliefs}

We measured individuals beliefs about what important or valued others think about doing presented tasks with three items: "Most people who matter to me would approve my doing this intervention.", "Most people who matter to me would think that I should do this intervention", "Most people who matter to me would think it's a good idea to do this intervention". Participants responded to items on a 7-point scale ranging from "completely disagree" to "completely agree" $(\alpha=.86)$.

\subsubsection{Self-control Beliefs}

We assessed participants beliefs about being able to effectively cope and handle the task demands with three items: "Performing this intervention would be very easy for me", "If I wanted, I could do it without any problem", "This activity wouldn't cause any trouble to me". Participants responded to items on a 7-point scale ranging from "completely disagree" to "completely agree" $(\alpha=.96)$.

\subsubsection{Behavioral Intention}

We measured intention to continue the positive intervention with a scale consisting of three items: "In the next week I intend to try out the proposed intervention", "In the next week I intend to perform the proposed intervention", "In the next week I intend to check out the proposed intervention". Participants responded to items on a 7-point scale ranging from "completely disagree" to "completely agree" $(\alpha=.95)$. 


\subsection{Gratitude Expression via Texting}

To account for the laboratory settings of the experiment, we developed a brief interpersonal intervention based on the Gratitude Letter exercise (Seligman et al. 2005). Rather than writing a note to the benefactor and present it face-to-face, we asked each participant to express their gratitude to their benefactor by sending a text message (Gratitude Texting).

Think of a person to whom you are grateful for something. If possible, it should be a person to whom you rarely express gratitude or have never thanked him or her before. Consider what you would like to write to this person. May this be something personally important to you, or perhaps something that you have not thanked yet for, or you feel that you have not thanked enough. Soon you will be asked to send this person a text message expressing your gratitude for this very thing. Let your SMS consists of at least 10-15 words. You will have three minutes to think about its content.

After three minutes of preparation, participants received further instruction:

Turn off airplane mode on your phone. Write and send an SMS to the person of your choice containing an expression of gratitude. After sending the SMS, switch your phone back to airplane mode.

This intervention involved the core elements of the gratitude expression: identifying a good event, appreciation of the event, acknowledging the role of the benefactor in producing the positive outcome, and the actual act of communicating gratitude. Noteworthy, this intervention did not account for the feedback from the benefactor, because it was not possible to control the time when the feedback (the return text) arrived.

For the control condition, participants were asked to send a neutral text message to their acquaintance without any constraints on the topic. This condition allowed accounting for any physiological and subjective responses related to texting in general (Lin and Peper 2009). The instructions were:

Soon you will be asked to send to a person of your choice a text message with a neutral (e.g., informative) content. You will have three minutes to think about its content. Turn off airplane mode on your phone. Write and send an SMS to the person of your choice containing what you have prepared. After sending the SMS, switch your phone back to airplane mode.

For the second text message participants received instruction that they can send it to the same or different person than the first text message.

\subsection{Daily Gratitude Intervention}

To account for daily gratitude expression training, we informed participants about an opportunity to continue a similar gratitude routine at home (Seligman et al. 2005; Kaczmarek et al. 2015). This intervention was a modified version of the Letter of Gratitude and was introduced in the following way:

In recent years, psychologists have established that there are exercises that can increase well-being and happiness. One of them is the "Letter of Gratitude." This exercise consists of writing a letter or a message of gratitude to someone. The note is sent to the addressee via text message, e-mail, or traditional mail. The exercise involves writing three letters of gratitude, each at an interval of one week. 
After reading the instruction, participants reported their motivation (utility beliefs, social norm, and self-efficacy beliefs) and behavioral intention to perform the intervention in daily life for 21 days. Their activity was reported via e-mail after three weeks.

\subsection{Physiological Reactivity}

Raw physiological data consisted of values averaged for each $30 \mathrm{~s}$ epochs. These data had $5.5 \%$ of random missing values due to occasional technical issues during the laboratory part of the study, causing artifacts in the physiological recording. We calculated average values for two moments for each gratitude/neutral message: (1) the final minute of taskpreceding baseline; (2) preparing text messages. Next, we calculated reactivity scores for HR, CO, and TPR, where the score of the task-preceding baseline was subtracted from the text message preparation period. Finally, we calculated gratitude reactivity by excluding reactivity to interpersonal communication via text messages, where we subtracted reactivity to preparing neutral text messages from the reactivity to preparing gratitude text messages. These corrected values were later used in the path model. Similarly, we subtracted self-reports of challenge and threat evaluations to the neutral text message from gratitude text message evaluations. Higher scores indicated more intense responses to gratitude expression.

\subsection{Analytical Strategy}

\subsubsection{Preliminary Analysis}

Before testing the main hypothesis, we checked whether participants in our study displayed a physiological engagement in preparing text messages. We compared task-preceding baseline cardiovascular values with message preparation periods. The biopsychosocial model of challenge and threat assumes that the individuals must be physiologically engaged in the task to examine the occurrence of challenge and threat states. HR is considered as the marker of motivational readiness, where increased HR is interpreted as an increase in physiological engagement.

\subsubsection{Main Analysis}

We used path analysis to test if behavior (exercising gratitude expression in daily life) was predicted by behavioral intention, intention by motivations, motivations by physiological reactivity and evaluations to gratitude expression observed in the laboratory, and if physiological reactivity and evaluations were predicted by individual differences in trait gratitude, depressiveness, and gender. We accounted for CO and TPR as indicators of challenge and threat cardiovascular patterns predictive of behavioral outcomes in goal-oriented actions (Behnke and Kaczmarek 2018; Hase et al. 2018). The weighted least-square with mean and variance correction estimator (WLSMV) was used to evaluate the fit of the model with the binary outcome (did the person continued the gratitude intervention in daily life or not) (Muthén and Muthén 2012). In line with the theoretical model and previous structural equation models examining theory of planned behavior (Ajzen 1991; Hoyt et al. 2009; Kaczmarek et al. 2014a), the residual variance of variables related to participants' motivation was freed to correlate amongst each other. Path analysis was conducted using mPlus 7.2 package (Muthén and Muthén 2012). To evaluate the fit of the model we calculated 
RMSEA (root mean square error of approximation), which is the recommended fit index for the WLSMV estimator, with values <.06 indicating good fit (Yu and Muthén 2002), WRMR (weighted root mean square residual), which is a recommended alternative to SRMR (standardized root mean square residual) for models with categorical outcome, with values $<1.0$ indicative of good fit, and CFI (confirmatory fit index) with values above .90 indicating acceptable models (Bentler 1990).

\section{Results}

\subsection{Preliminary Analysis}

Descriptive statistics of challenge and threat physiological responses during preparation of gratitude and neutral text messages are presented in Table 2. Participants displayed higher HR during message preparation relative to baseline for gratitude, $t(181)=-4.167$, $p<.001, d=0.31$, and neutral, $t(182)=-3.799, p<.001, d=0.29$, messages. This indicates that both tasks significantly motivated participants to action-readiness as a requirement for further challenge/threat evaluations and vascular responses (Blascovich 2008). Participants also displayed higher $\mathrm{CO}$ during the tasks relative to baseline for gratitude, $t(177)=-4.68, p<.001, d=0.35$, and neutral, $t(178)=-4.13, p<.001, d=0.31$, condition. We also observed increased TPR during gratitude, $t(177)=-2.71, p=.007, d=0.18$, and neutral, $t(178)=-2.38, p=.02, d=0.20$, condition relative to the baseline. This supports the expectation that the text messaging task influenced cardiovascular processes relevant for the study of situational challenge and threat.

\subsection{Main Analysis}

Descriptive statistics and correlations are presented in Table 3. Of the 196 participants, 84 responded to our follow-up e-mail. Out of these, 15 declared that they continued the

Table 2 Evaluations and physiological markers of challenge and threat states to sending text messages

\begin{tabular}{|c|c|c|c|c|}
\hline \multirow[t]{2}{*}{ Parameter } & \multicolumn{2}{|c|}{ Neutral condition } & \multicolumn{2}{|c|}{$\begin{array}{l}\text { Gratitude condi- } \\
\text { tion }\end{array}$} \\
\hline & $M$ & $S D$ & $M$ & $S D$ \\
\hline TPR baseline & 1.03 & 0.36 & 1.04 & 0.37 \\
\hline TPR task & 1.05 & 0.36 & 1.06 & 0.36 \\
\hline CO baseline & 5.57 & 1.21 & 5.55 & 1.22 \\
\hline CO task & 5.68 & 1.20 & 5.69 & 1.23 \\
\hline HR baseline & 79.1 & 10 & 77.8 & 10 \\
\hline HR task & 80.23 & 10 & 79.9 & 10 \\
\hline Challenge evaluation & 33.00 & 7.00 & 35.00 & 7.00 \\
\hline Threat evaluation & 25.00 & 11.00 & 28.00 & 11.00 \\
\hline
\end{tabular}

$T P R$ Total peripheral resistance, $C O$ cardiac output, $H R$ heart rate. Units: $\mathrm{TPR}=\mathrm{mmHg} \mathrm{s} \mathrm{ml}{ }^{-1} ; \mathrm{CO}=\mathrm{L} \mathrm{min}^{-1} ; \mathrm{HR}=$ beats per minute

All differences between corresponding baseline and task recordings were significant at $p<.05$ 


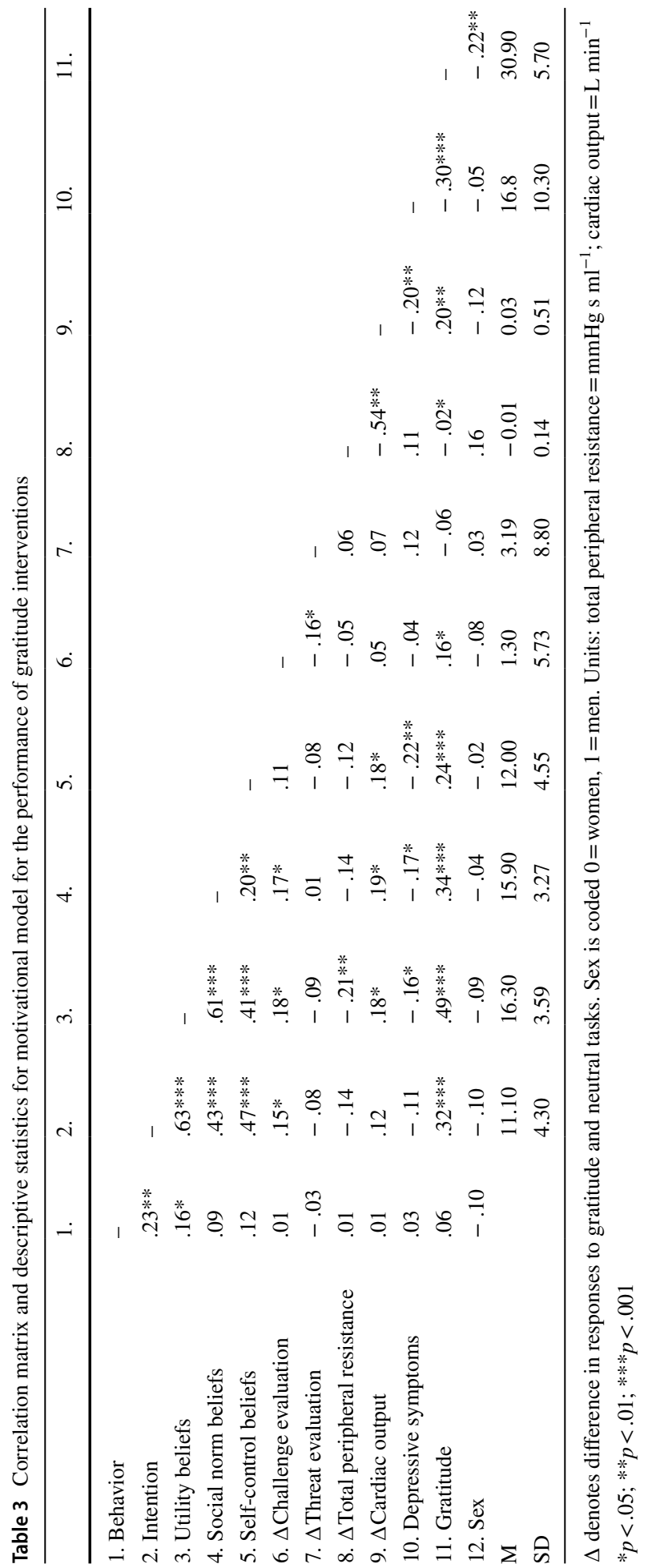


Gratitude Texting intervention. In line with the biopsychosocial model of challenge and threat (Blascovich and Mendes 2000), we considered introducing challenge and threat evaluations as predictors of physiological responses. However, challenge/threat evaluations and physiological responses were not correlated witch each other $(p s>.05)$. Thus, we introduced the physiological and cognitive components as parallel predictors of motivation. The mediational path model is presented in Fig. 1. This model fit the data at acceptable level $\chi^{2}$ $(54)=73.79, p=0.04$, RMSEA $=.04,90 \%$ CI $[.01, .07]$, WRMR $=0.81$, CFI $=.92$. Insignificant paths had no effect on model fit, $\Delta \chi^{2}(17)=23.46, p=.135$, and were removed.

As expected, individuals with stronger intentions to complete the gratitude intervention in daily life were more likely to complete it, $\beta=0.45, p=.002$. Individuals displayed stronger intention to complete the gratitude intervention when they had high utility beliefs, $\beta=0.54, p<.001$ and self-control beliefs, $\beta=0.26, p<.001$.

Individuals that reacted with increased TPR to gratitude expression via text message displayed lower motivation to complete the future intervention characterized by lower utility beliefs, $\beta=-0.89, p<.001$, lower social norms, $\beta=-0.65, p<.001$, and lower self-control beliefs, $\beta=-0.44, p<.001$. Individuals with lower trait gratitude reacted to the gratitude expression with increased TPR, $\beta=-0.60, p<.001$, and decreased CO, $\beta=0.25, p<.001$. Also, individuals with more depressive symptoms reacted to the task with increased TPR, $\beta=0.25, p=.001$, and decreased $\mathrm{CO}, \beta=-0.23, p<.001$. Individuals with higher trait gratitude evaluate gratitude expression task as a stronger challenge, $\beta=0.22, p=.001$.

We observed significant indirect effects in the model. First, individuals with higher trait gratitude were more likely to complete the intervention, $\beta=0.16, p=.006$, via lower TPR reactivity to gratitude text message, stronger utility beliefs, self-control beliefs, and intention to initiate the gratitude intervention in daily life, $p s<.05$. Second, individuals with less depressive symptoms were more likely to complete the intervention, $\beta=-0.07, p=.020$, also via lower TPR reactivity, stronger utility beliefs, self-control beliefs, and intention, $p s<.05$.

Women reported higher trait gratitude, $\beta=-0.23, p=.006$, but not depression compared to men. There was no significant indirect effect of sex on behavioral outcomes. However, women endorsed stronger intentions to initiate the intervention as indicated by a significant indirect effect, $\beta=-0.08, p<.05$. This indirect effect operated via higher gratitude, TPR

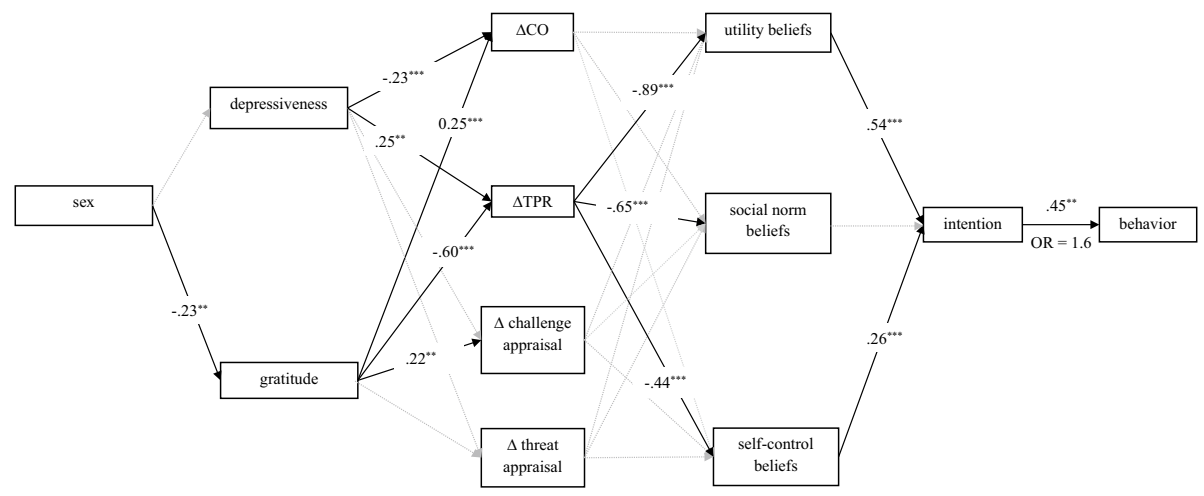

Fig. 1 A motivational model for the performance of gratitude interventions. Note: Non-significant paths are in grey. All coefficients are standardized. $\Delta$ denotes difference in responses to gratitude and neutral tasks. CO, cardiac output; TPR, total peripheral resistance; behavior = gratitude intervention completion in daily life; $\mathrm{OR}=$ odds ratio. Behavior is coded $0=$ no, $1=$ yes. Sex is coded $0=$ women, $1=$ men. ${ }^{*} p<.05$, $* * p<.01, * * * p<.001$ 
reactivity, and utility beliefs, $\beta=-0.07, p<.05$, and via higher gratitude, TPR reactivity, and self-control beliefs, $\beta=-0.02, p<.05$.

\section{Discussion}

In this study, we examined cardiovascular responses to a brief gratitude expression trial and their predictive role in completing a three-week gratitude intervention. We examined whether challenge and threat responses predicted further motivations, behavioral intentions, and subsequent daily actions related to gratitude expression in daily life. Besides situational factors, we emphasized the role of individual differences that facilitate motivations towards gratitude-related behaviors, i.e., gratitude, depressive symptoms, and sex-related normative beliefs. We provided high ecological validity to the study by inviting participants to perform the intervention on their own for three weeks after the laboratory session. We found that personality differentiated physiological responding to gratitude expression and that these reactions predicted gratitude intervention completion.

We found that challenge/threat cardiovascular reactivity (TPR reactivity) had a direct effect on motivations and an indirect effect on completing the gratitude intervention. Individuals that responded with increased TPR reactivity to the gratitude expression trial were less likely to perceive the gratitude intervention as valuable (lower utility beliefs). They also reported lower belief in their ability to perform the intervention and perceived more negative social norms regarding the intervention. This, in turn, produced a weaker intention to initiate the intervention and decreased the likelihood of performing the intervention in daily life. These findings indicate that TPR reactivity is an important physiological marker in the assessment of person-activity fit. This might result from the fact that increased TPR impedes efficient energy mobilization (Seery 2011). Increased TPR is also characteristic of the experience of negative emotions such as anger, embarrassment, or sadness (Kreibig 2010). Challenge and threat states are also characterized by CO reactivity, however, we found no influence of $\mathrm{CO}$ on motivation, intention, or task completion. This is an unexpected finding given that $\mathrm{CO}$ reactivity has been related to depression in previous studies (Phillips 2011).

We observed that physiological indexes and subjective measures of challenge and threat states were unrelated. This indicates that evaluations and physiological responses to challenge and threat operated in separation. Although this finding contradicts the theory (Blascovich 2008), it is in line with some previous studies that revealed a non-significant relationship between CO and TPR, and challenge/threat evaluation (Behnke et al. 2020; Brimmell et al. 2019). This might result from the fact that physiological responses are mainly influenced by the homeostatic mechanisms and depend on several factors with stress appraisal being only one of them (Levenson 2014). Moreover, for technical reasons, participants completed self-reports before the tasks, relying on their expectations for the task, while physiological measures were recorded during the actual task. This might provide more predictive validity to the physiological measures relative to self-reports. Data from aggregated continuous physiological recordings are more sensitive than single-point self-reports and are not biased by prospective or retrospective distortions that can occur with self-reports (Blascovich et al. 2011).

We found indirect effects for trait gratitude and frequency of depressive symptoms on continuing intervention in daily life. Both effects were mediated by TPR reactivity, utility and self-control beliefs, and intention. Extending previous findings, we found that 
individuals with low trait gratitude and high depressiveness were more likely to experience threat state to gratitude expression and, in turn, expect negative responses from others about taking part in gratitude activities in daily life (social norm beliefs). Those individuals were also more likely to view the gratitude intervention as useless and believed less in their self-efficacy for the intervention. These findings offer insight into mechanisms behind the motivation to complete gratitude interventions. Moreover, we observed that individual differences in trait gratitude and depressiveness affected challenge and threat responses to gratitude expression task at both cognitive and physiological levels. As expected, participants with higher trait gratitude were more likely to respond to gratitude expression task with challenge evaluation and challenge cardiovascular response (decreased TPR and increased $\mathrm{CO}$ ). In contrast, participants reporting more depressive symptoms reacted to gratitude expression task with threat cardiovascular response (increased TPR and decreased $\mathrm{CO}$ ).

Our findings complement previous work regarding the theory of planned behavior (Ajzen 1991, 2011) as an explanatory framework for the study of motivation to gratitude interventions' performance (Kaczmarek et al. 2013, 2014a, b, 2015). We believe that his theory might be considered an alternative approach towards the assessment of personactivity fit to other methods present in the literature (Lyubomirsky and Layous 2013). The advantage of using Ajzen's approach towards motivation, intention, and action, is that by using this theory it becomes possible to integrate positive psychological interventions literature with a broader framework of planned or intentional activity (Ajzen 1991, 2011). We replicated previous findings where motivational components predicted intention and subsequently self-initiation of positive interventions. Participants that reported low intention were less likely to continue intervention in daily life. The percentage of individuals performing the intervention in daily life was low (8\%) in line with results from previous studies (e.g., Kaczmarek et al. 2013, 2015). This points out to low effectiveness of positive interventions despite their high efficacy (Hone et al. 2015). Gratitude interventions can be considered still much more feasible than complex healthy initiatives (Madden et al. 1992).

We found no sex differences in the likelihood to participate in gratitude interventions (Kaczmarek et al. 2013, 2015). However, we observed that women maintained stronger intentions to start the intervention. These two findings seem to suggest that women needed stronger motivation to achieve the same behavioral outcome as men. Stronger motivation among women was fueled by their higher levels of trait gratitude, more moderate physiological responding, greater expectation of benefits from the intervention, and greater ease of its execution. These findings corroborate previous findings that indicate a stronger preference for gratitude interventions among women. These findings also seem to point to a new phenomenon in the gratitude interventions literature, i.e., individual differences in motivational strength required to turn motivations into action. Thus, these findings correspond with the more general problem of the intention-behavior gap (e.g., Sheeran and Webb 2016).

\subsection{Limitations}

There are several limitations to this study. First, we examined students, which restricts generalization of the results to individuals of other age and education. Second, the gratitude texting intervention accounted for the expression of gratitude but not for the feedback from the benefactor as it would be the case in natural settings. Studying responses to gratitude feedback would provide more information on how individuals process gratitude in daily 
life, and which part of the two-step process (sending messages and receiving feedback) is the most demanding. Third, participants received three minutes to develop the message. Thus, participants' responses might have been influenced by time-pressure; a factor that elicits stress (Sharma and McKenna 2001; Moorthy et al. 2003). Other challenge and threat patterns might occur if participants had less stringent conditions for contemplating the target and the content of the grateful message. Finally, despite quantitative evidence for the validity of the gratitude texting intervention (as presented in the manipulation check), we had little control over the actual content of the messages due to privacy reasons.

\subsection{Practical Implications}

Due to its experimental design and the daily life follow-up, this study has some practical implications. First, we provide new evidence that emphasizes the need for evaluation of person-activity fit before intervention administration (Lyubomirsky and Layous 2013). This is the first study to indicate that the person-activity mismatch is observable on a physiological level. This might indicate that potential interventions' recipients might be instructed to observe their physiological responses as a supplementary source of information whether this specific intervention fits their preferences. As physiological responses were related to behavioral outcomes, we might expect that such observational strategy focused on "gut feelings" might reduce the odds of investing into an activity that will lead to a failure; an outcome that might be exceptionally harmful for depressed individuals who tend to have low self-efficacy beliefs in general (Luszczynska et al. 2005). Second, we indicated that individuals with high trait-gratitude were more likely to engage in the gratitude intervention. Although this finding seems straightforward, it suggests that individuals might capitalize on the use of their signature strengths rather than aiming to develop their secondary strengths (Seligman et al. 2005). Finally, building upon prior research on emotions and somatic health (Pressman and Cohen 2005), our findings suggest that individuals' cardiovascular health is at risk when they face a gratitude intervention that does not fit their personality. This may be particularly problematic for intervention recipients with high cardiovascular risk. This implication is important because gratitude interventions have been targeted at patients with acute cardiovascular disease (Huffman et al. 2011). Findings in our study suggest that cardiovascular patients' depression and trait-gratitude should be assessed before encouraging them to perform gratitude interventions.

\section{Conclusion}

The strength of this study is that we proposed a novel, in-depth way to study the personactivity fit. Using a brief gratitude expression trial along with an explicit focus on physiological responding is a promising strategy to reduce the risk of intervention mismatch. As the positive psychological interventions literature evolves, it becomes increasingly possible to move from the "one-size-fits-all" approach towards development of interventions that are challenging yet do not impose a threat on vulnerable recipients.

Author's Contribution JE and LDK: study design, data analysis, writing the manuscript; MB: carrying out the experiment, writing the manuscript, MD, MK: carrying out the experiment. 
Funding This project was Funded by the National Science Center, Poland (UMO- 2014-15/N/HS6/04151). Research grant awarded to JE as the Principal Investigator. Preparation of this article was supported by doctoral scholarships from National Science Centre in Poland (UMO-2019/32/T/HS6/00039) and Adam Mickiewicz University Foundation to MB.

\section{Compliance with Ethical Standards}

Conflict of interest The authors declare that they have no conflict of interest.

Informed Consent All participants provided written informed consent. The study was accepted by the Institutional Ethics Committee.

Open Access This article is licensed under a Creative Commons Attribution 4.0 International License, which permits use, sharing, adaptation, distribution and reproduction in any medium or format, as long as you give appropriate credit to the original author(s) and the source, provide a link to the Creative Commons licence, and indicate if changes were made. The images or other third party material in this article are included in the article's Creative Commons licence, unless indicated otherwise in a credit line to the material. If material is not included in the article's Creative Commons licence and your intended use is not permitted by statutory regulation or exceeds the permitted use, you will need to obtain permission directly from the copyright holder. To view a copy of this licence, visit http://creativecommons.org/licenses/by/4.0/.

\section{References}

Ajzen, I. (1991). The theory of planned behavior. Organizational Behavior and Human Decision Processes, $50,179-211$.

Ajzen, I. (2011). The theory of planned behavior: reactions and reflections. Psychology and Health, 26, 1113-1127.

Becker, J. A., \& Smenner, P. C. (1986). The spontaneous use of thank you by preschoolers as a function of sex, socioeconomic status, and listener status. Language in Society, 15, 537-545.

Behnke, M., \& Kaczmarek, L. D. (2018). Successful performance and cardiovascular markers of challenge and threat: A meta-analysis. International Journal of Psychophysiology, 130, 73-79.

Behnke, M., Kosakowski, M., \& Kaczmarek, L. D. (2020). Social challenge and threat predict performance and cardiovascular responses during competitive video gaming. Psychology of Sport and Exercise, 46, 101584.

Bentler, P. M. (1990). Comparative fit indexes in structural models. Psychological Bulletin, 107, 238.

Beutel, A. M., \& Marini, M. M. (1995). Gender and values. American Sociological Review, 60, 436-448.

Blascovich, J. (2008). Challenge and threat. In A. J. Elliot (Ed.), Handbook of approach and avoidance motivation (pp. 431-445). New York, NY: Psychology Press.

Blascovich, J., \& Mendes, W. B. (2000). Challenge and threat appraisals: The role of affective cues. In J. Forgas (Ed.), Feeling and thinking: The role of affect in social cognition (pp. 59-82). New York, NY: Cambridge University Press.

Blascovich, J., Mendes, W. B., Vanman, E., \& Dickerson, S. (2011). Social psychophysiology for social and personality psychology. Thousand Oaks: Affective Sciences Series, SAGE.

Bolier, L., Haverman, M., Westerhof, G. J., Riper, H., Smit, F., \& Bohlmeijer, E. (2013). Positive psychology interventions: a meta-analysis of randomized controlled studies. BMC Public Health, 13, 119.

Brimmell, J., Parker, J., Wilson, M. R., Vine, S. J., \& Moore, L. J. (2019). Challenge and threat states, performance, and attentional control during a pressurized soccer penalty task. Sport, Exercise, and Performance Psychology, 8, 63-79.

Bromnick, R. D., \& Swallow, B. L. (2001). Parties, Lads, Friends, Love and Newcastle United: a study of young people's values. Educational Studies, 27, 143-158.

Brownley, K. A., Hurwitz, B. E., \& Schneiderman, N. (2000). Cardiovascular psychophysiology. In J. T. Cacioppo, L. G. Tassinary, \& G. G. Berntson (Eds.), Handbook of psychophysiology (pp. 224-264). New York, NY: Cambridge University Press. 
Chakhssi, F., Kraiss, J. T., Sommers-Spijkerman, M., \& Bohlmeijer, E. T. (2018). The effect of positive psychology interventions on well-being and distress in clinical samples with psychiatric or somatic disorders: a systematic review and meta-analysis. BMC Psychiatry, 18, 211.

Drążkowski, D., Kaczmarek, Ł. D., \& Kashdan, T. B. (2017). Gratitude pays: A weekly gratitude intervention influences monetary decisions, physiological responses, and emotional experiences during a trustrelated social interaction. Personality and Individual Differences, 110, 148-153.

Emmons, R. A., \& McCullough, M. E. (2003). Counting blessings versus burdens: An experimental investigation of gratitude and subjective well-being in daily life. Journal of Personality and Social Psychology, 84, 377-389.

Francis, J.J., Eccles, M.P., Johnston, M., Walker, A.E., Grimshaw, J.M., Foy, R., et al. (2004). Constructing questionnaires based on the theory of planned behaviour. A manual for health services researchers. In: Centre for Health Services Research, University of Newcastle Upon Tyne, UK.

Froh, J. J., Kashdan, T. B., Ozimkowski, K. M., \& Miller, N. (2009). Who benefits the most from a gratitude intervention in children and adolescents? Examining positive affect as a moderator. The Journal of Positive Psychology, 4, 408-422.

Gordon, A. K., Musher-Eizenman, D. R., Holub, S. C., \& Dalrymple, J. (2004). What are children thankful for? An archival analysis of gratitude before and after the attacks of September 11. Applied Developmental Psychology, 25, 541-553.

Hase, A., O’Brien, J., Moore, L. J., \& Freeman, P. (2018). The relationship between challenge and threat states and performance: A systematic review. Sport, Exercise, and Performance Psychology, 8, $123-144$.

Hendriks, T., Schotanus-Dijkstra, M., Hassankhan, A., Graafsma, T., Bohlmeijer, E., \& de Jong, J. (2018a). The efficacy of positive psychology interventions from non-Western countries: A systematic review and meta-analysis. International Journal of Wellbeing, 8, 71-98.

Hendriks, T., Schotanus-Dijkstra, M., Hassankhan, A., Graafsma, T., \& de Jong, J. E. T. (2018a). The efficacy of multi-component positive psychology interventions: A systematic review and meta-analysis. In: T. Hendriks (Ed.), Positive psychology interventions in a multi-ethnic and cross-cultural context (Doctoral thesis). Amsterdam: University of Amsterdam.

Hone, L. L. C., Jarden, A., \& Schofield, G. M. (2015). An evaluation of positive psychology intervention effectiveness trials using the RE-AIM framework: A practice-friendly review. The Journal of Positive Psychology, 10, 303-322.

Hoyt, A. L., Rhodes, R. E., Hausenblas, H. A., \& Giacobbi, P. R., Jr. (2009). Integrating five-factor model facet-level traits with the theory of planned behavior and exercise. Psychology of Sport and Exercise, 10, 565-572.

Huffman, J. C., DuBois, C. M., Healy, B. C., Boehm, J. K., Kashdan, T. B., Celano, C. M., et al. (2014). Feasibility and utility of positive psychology exercises for suicidal inpatients. General Hospital Psychiatry, 36, 88-94.

Huffman, J. C., Mastromauro, C. A., Boehm, J. K., Seabrook, R., Fricchione, G. L., Denninger, J. W., \& Lyubomirsky, S. (2011). Development of a positive psychology intervention for patients with acute cardiovascular disease. Heart International, 6, 47-53.

Jackowska, M., Brown, J., Ronaldson, A., \& Steptoe, A. (2016). The impact of a brief gratitude intervention on subjective well-being, biology and sleep. Journal of Health Psychology, 21, 2207-2217.

Kaczmarek, L. D., Goodman, F. R., Drążkowski, D., Kashdan, T. B., Połatyńska, K., \& Komorek, J. (2014a). Instructional support decreases desirability and initiation of a gratitude intervention. Personality and Individual Differences, 64, 89-93.

Kaczmarek, L. D., Kashdan, T. B., Drążkowski, D., Bujacz, A., \& Goodman, F. (2014b). Why do greater curiosity and fewer depressive symptoms predict gratitude intervention use? Desirability, social norm beliefs, and perceived self-control. Personality and Individual Differences, 66, 165-170.

Kaczmarek, L. D., Kashdan, T. B., Drążkowski, D., Enko, J., Kosakowski, M., Szäefer, A., et al. (2015). Why people prefer gratitude journals over gratitude letters. A differentiation of motivations towards individual and interpersonal gratitude interventions. Personality and Individual Differences, 75, $1-6$.

Kaczmarek, L. D., Kashdan, T. B., Kleiman, E., Bączkowski, B., Enko, B., Siebers, A., et al. (2013). Who self-initiates gratitude interventions in daily life? An examination of intentions, curiosity, depressive symptoms, and life satisfaction. Personality and Individual Differences, 55, 805-810.

Kashdan, T. B., Mishra, A., Breen, W. E., \& Froh, J. J. (2009). Gender differences in gratitude: Examining appraisals, narratives, the willingness to express emotions, and changes in psychological needs. Journal of Personality, 77, 691-730.

Krause, N. (2006). Gratitude toward God, stress, and health in late life. Research on Aging, 28, $163-183$. 
Kreibig, S. D. (2010). Autonomic nervous system activity in emotion: A review. Biological Psychology, 84, 394-421.

Lambert, N. M., Clark, M. S., Durtschi, J., Fincham, F. D., \& Graham, S. M. (2010). Benefits of expressing gratitude: Expressing gratitude to a partner changes one's view of the relationship. Psychological Science, 21, 574-580.

Lazarus, R. S., \& Folkman, S. (1984). Stress. New York: Appraisal and Coping.

Levenson, R. W. (2014). The autonomic nervous system and emotion. Emotion Review, 6, 100-112.

Lin, I., \& Peper, E. (2009). Psychophysiological patterns during cell phone text messaging: A preliminary study. Applied Psychophysiology and Biofeedback, 34, 53-57.

Lovallo, W. R., \& Gerin, W. (2003). Psychophysiological reactivity: Mechanisms and pathways to cardiovascular disease. Psychosomatic Medicine, 65, 36-45.

Luszczynska, A., Scholz, U., \& Schwarzer, R. (2005). The general self-efficacy scale: Multicultural validation studies. The Journal of Psychology, 139, 439-457.

Lyubomirsky, S., Dickerhoof, R., Boehm, J. K., \& Sheldon, K. M. (2011). Becoming happier takes both a will and a proper way: An experimental longitudinal intervention to boost well-being. Emotion, 11, 391-402.

Lyubomirsky, S., \& Layous, K. (2013). How do simple positive activities increase well-being? Current Directions in Psychological Science, 22, 57-62.

Madden, T. J., Ellen, P. S., \& Ajzen, I. (1992). A comparison of the theory of planned behavior and the theory of reasoned action. Personality and Social Psychology Bulletin, 18, 3-9.

McCullough, M. E., Emmons, R. A., \& Tsang, J. A. (2002). The grateful disposition: a conceptual and empirical topography. Journal of Personality and Social Psychology, 82, 112-127.

Mendes, W. B., Blascovich, J., Hunter, S., Lickel, B., \& Jost, J. (2007). Threatened by the unexpected: Physiological responses during social interactions with expectancy-violating partners. Journal of Personality and Social Psychology, 92, 698-716.

Mendes, W. B., Major, B., McCoy, S., \& Blascovich, J. (2008). How attributional ambiguity shapes physiological and emotional responses to social rejection and acceptance. Journal of Personality and Social Psychology, 94, 278-291.

Mendes, W. B., \& Park, J. (2014). Chapter six-neurobiological concomitants of motivational states. Advances in Motivation Science, 1, 233-270.

Michie, S., Ashford, S., Sniehotta, F. F., Dombrowski, S. U., Bishop, A., \& French, D. P. (2011). A refined taxonomy of behaviour change techniques to help people change their physical activity and healthy eating behaviours: The CALO-RE taxonomy. Psychology and Health, 26(11), 1479-1498.

Misiak, M., Kaczmarek, L. D., Kashdan, T. B., Zimny, M., Urbański, M., Zasiński, A., et al. (2015). Prenatal exposure to sex hormones predicts gratitude intervention use. Examination of digit ratio, motivational beliefs, and online activities. Personality and Individual Differences, 77, 68-73.

Moore, L. J., Vine, S. J., Wilson, M. R., \& Freeman, P. (2014). Examining the antecedents of challenge and threat states: The influence of perceived required effort and support availability. International Journal of Psychophysiology, 93, 267-273.

Moorthy, K., Munz, Y., Dosis, A., Bann, S., \& Darzi, A. (2003). The effect of stress-inducing conditions on the performance of a laparoscopic task. Surgical Endoscopy and Other Interventional Techniques, 17, 1481-1484.

Muthén, L. K., \& Muthén, B. O. (2012). Mplus user's guide (7th ed.). Los Angeles, CA: Muthén \& Muthén.

Parks, A. C., Della Porta, M. D., Pierce, R. S., Zilca, R., \& Lyubomirsky, S. (2012). Pursuing happiness in everyday life: The characteristics and behaviors of online happiness seekers. Emotion, 12, 1222-1234.

Penaz, J. (1973). Photoelectric measurement of blood pressure, volume and flow in the finger. In Paper presented at the digest of the 10th international conference on medical and biological engineering. Dresden.

Peterson, C., \& Seligman, M. E. P. (2004). Character strengths and virtues: A handbook and classification. Washington, DC: APA Press.

Phillips, A. C. (2011). Blunted cardiovascular reactivity relates to depression, obesity, and self-reported health. Biological Psychology, 86, 106-113.

Pressman, S., \& Cohen, Sh. (2005). Does Positive Affect Influence Health? Psychological Bulletin, 131, 925-971.

Radloff, L. S. (1977). The CES-D scale: A self-report depression scale for research in the general population. Applied Psychological Measurement, 1, 385-401.

Redwine, L. S., Henry, B. L., Pung, M. A., Wilson, K., Chinh, K., Knight, B., et al. (2016). Pilot randomized study of a gratitude journaling intervention on heart rate variability and inflammatory biomarkers in patients with stage B heart failure. Psychosomatic Medicine, 78, 667-676. 
Seery, M. (2011). Challenge or threat? Cardiovascular indexes of resilience and vulnerability to potential stress in humans. Neuroscience and Biobehavioral Reviews, 35, 1603-1610.

Seligman, M. E. P., Steen, T. A., Park, N., \& Peterson, C. (2005). Positive psychology progress: Empirical validation of interventions. American Psychologist, 60, 410-421.

Senf, K., \& Liau, A. K. (2013). The effects of positive interventions on happiness and depressive symptoms, with an examination of personality as a moderator. Journal of Happiness Studies: An Interdisciplinary Forum on Subjective Well-Being, 14(2), 591-612.

Sharma, D., \& McKenna, F. P. (2001). The role of time pressure on the emotional Stroop task. British Journal of Psychology, 92, 471-481.

Sheeran, P., \& Webb, T. L. (2016). The intention-behavior gap. Social and Personality Psychology Compass, $10(9), 503-518$.

Sin, N. L., \& Lyubomirsky, S. (2009). Enhancing well-being and alleviating depressive symptoms with positive psychology interventions: A practice friendly meta-analysis. Journal of Clinical Psychology, 65, 467-487.

Skinner, N., \& Brewer, N. (2002). Dynamics of threat and challenge appraisals prior to stressful achievement events. Journal of Personality and Social Psychology, 83, 678-692.

vanOyen Witvliet, C., Knoll, R. W., Hinman, N. G., \& DeYoung, P. A. (2010). Compassion-focused reappraisal, benefit-focused reappraisal, and rumination after an interpersonal offense: Emotion-regulation implications for subjective emotion, linguistic responses, and physiology. The Journal of Positive Psychology, 5, 226-242.

Ventimiglia, J. C. (1982). Sex roles and chivalry: Some conditions of gratitude to altruism. Sex Roles, 8, 1107-1122.

Wood, A. M., Froh, J. J., \& Geraghty, A. W. (2010). Gratitude and well-being: A review and theoretical integration. Clinical Psychology Review, 30, 890-905.

Yu, C. Y. \& Muthén, B. (2002). Evaluation of model fit indices for latent variable models with categorical and continuous outcomes. In: Paper presented at the annual meeting of the American Educational Research Association, New Orleans, LA.

Publisher's Note Springer Nature remains neutral with regard to jurisdictional claims in published maps and institutional affiliations. 